\title{
Design of Wideband Power Amplifier in 130nm CMOS for LTE Applications
}

\author{
Faisal Abdul Hadi ${ }^{1}$, VeeraiyahThangasamy ${ }^{2}$, Shankar Duraikannan ${ }^{3}$ \\ ${ }^{1}$ School of Engineering, Asia Pacific University of Technology \& Innovation, Kuala Lumpur, Malaysia \\ faisal2691@hotmail.com \\ ${ }^{2}$ School of Engineering, Asia Pacific University of Technology \& Innovation, Kuala Lumpur, Malaysia \\ dr.veeraiyah@apu.edu.my \\ ${ }^{3}$ School of Engineering, Asia Pacific University of Technology \& Innovation, Kuala Lumpur, Malaysia \\ shankar@apu.edu.my
}

\begin{abstract}
Multiband power amplifier (PA) has become a crucial requirement for mobile communication systems that operate in variety bandwidth ranges along with different frequencies and output power. Replacement of more than two power amplifiers (PAs) by a single wideband PA is required to minimize power consumption and the chip size. This paper proposes, a wideband PA withfiltered matching network (FMN) to provide a wider bandwidth while improving linearity by harmonic suppression. The proposed matching network offers an operating bandwidth of $1.47 \mathrm{GHz}$ from the frequency $900 \mathrm{MHz}$ towards $2370 \mathrm{MHz}$, covering 24 LTE frequency bands. The frequency range is achieved at maximum output power of $27.5 \mathrm{dBm}$ with linear power of $26 \mathrm{dBm}$ at $1-\mathrm{dB}$ point. The ACLR value of $-34 \mathrm{dBc}$ is achieved at 1-dB output power which is within the requirement of LTE linearity specification.
\end{abstract}

Keyword - Adjacent channel leakage ratio (ACLR), bandwidth, filtered matching network (FMN), matching network, power amplifier, output power.

\section{INTRODUCTION}

With growing number of frequency bands in mobile communication to meet the demands of data rate, power amplifiers (PA) arerequired to operate at entire wide range of frequencies in mobile communication systems along with meeting the requirement of PAs linearity, adjacent channel leakage ratio (ACLR), error vector magnitude (EVM) and output power. However, existing multiple PAs operating for each frequency band would increase the complexity and cost of wireless communication systems especially when they are required to support more frequency bands [1]-[3]. In addition, PAs' distortions caused by their nonlinear behaviour, usually degrades their performance when high power and high efficiency is considered [1]-[3]. Therefore, removal of distortions caused by nonlinear behaviour of PA in diodes and transistors is crucial [1]-[3].In order to ensure broad coverage of frequencies by PA, a multimode multiband feature is required to be incorporated in its design to meet the communication standards of current and future mobile communication systems [1]-[4]. High output power and high efficiency with minimum distortions are achieved by incorporating a matching network in PA design.

The presence of matching network in PA design provides a maximized power transferred between source and load [1] [3] [4]. Power amplifier with optimized matching network ensures reduction of nonlinear signal generated by the PA. Proper design of matching network also improves PA performance for other parameters that include adjacent channel leakage ratio (ACLR) and error vector magnitude (EVM) [1]-[4].

Multiple power amplifiers have been in use for each frequency in fourth generation (4G) mobile phones. The power amplifiers are categorized to operate in two sets of frequency bands which include low frequencies of $700 / 800 / 850 / 900 \mathrm{MHz}$ and high frequencies of $1700 / 1800 / 1900 / 2500 / 2600 \mathrm{MHz}$ [1][5]. However, a single wideband power amplifier is demanded by PA market that can operate in multiple frequency bands simultaneously ranging from 700 to $3500 \mathrm{MHz}$ [1][4][5]. The expansion of power amplifier's bandwidth increases the chance of higher power consumption by PA in mobile phones which also depreciates the ACLR and EVM. The trending 4G technology in terms of Long Term Evolution (LTE) and LTE-Advanced (LTE-A) requires a power amplifier with wider bandwidth without degrading PA performance related to ACLR [1][3][8]-[13].

The data rate of mobile network is mainly influenced by transmission of power between the base stations and mobile communication systems. Thus the transmitted power plays a key role in providing high data rate which mainly depends on power amplifiers linearity [14]-[21]. On the other hand, the nonlinearity of the power amplifiers is contributed by the increase of peak to average power ratio (PAPR) of the modulating signals that are adopted to achieve high data rate through the mobile network [22]-[25]. Therefore, a power amplifier with wider bandwidth and improved efficiency requirements is ensured by incorporating an appropriate matching network [26]-[32]. This means that wide bandwidth of PA is required to be maintained while satisfying other parameters simultaneously. This paper presents an on-chip single wideband power amplifier through impedance 
matching network that is designed to operate on wide continuous range of frequencies between $900 \mathrm{MHz}$ to $2370 \mathrm{MHz}$.

\section{Methodology}

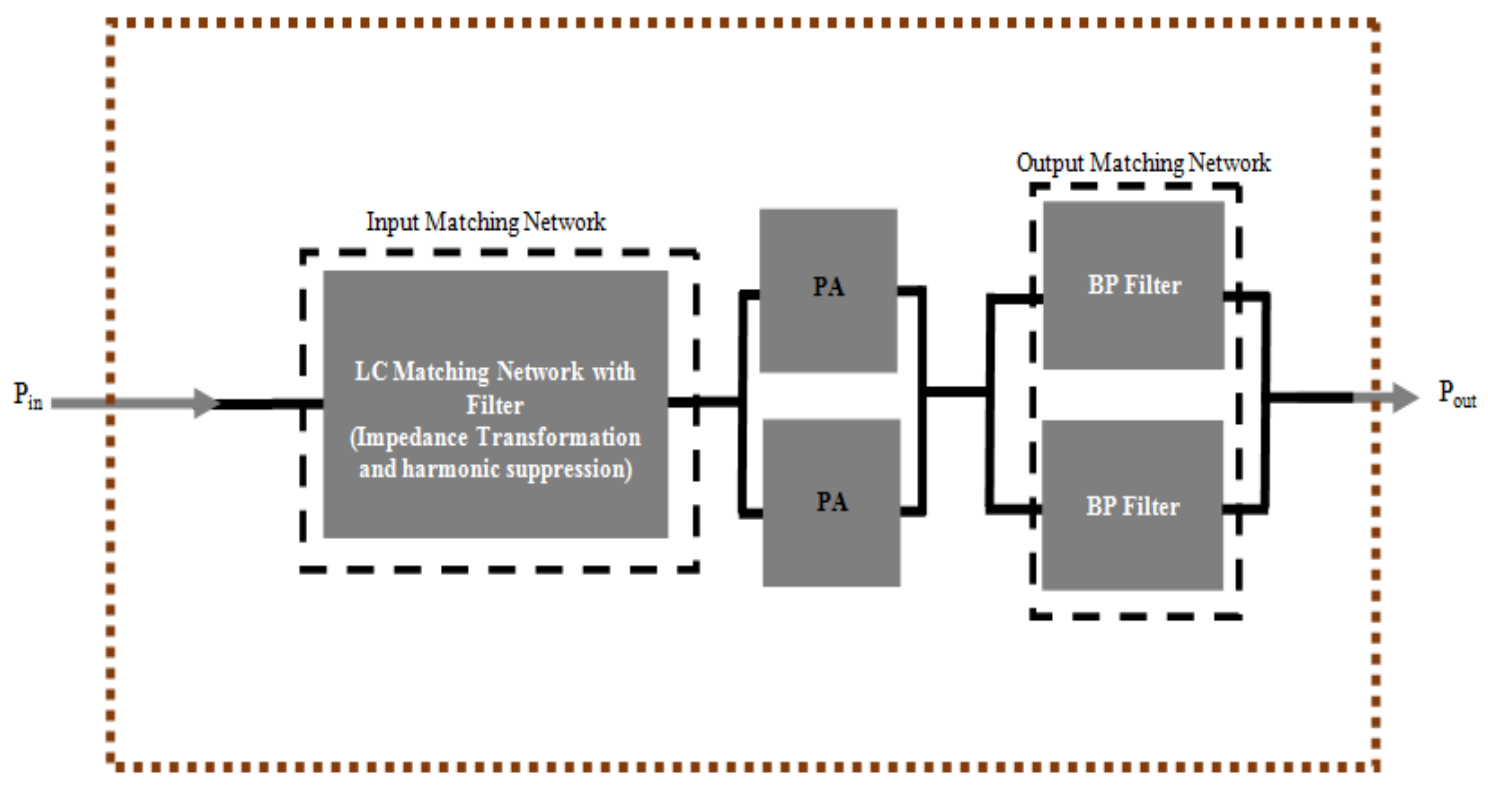

Fig 1. Circuit diagram of wideband PA with proposed matching network

The focus of this research is to design a wideband PA operating in frequencies from $900 \mathrm{MHz}$ to 2370MHz.These specifications comply with 3GPP standards of operating LTE/LTE-A throughout the world. In this research, the proposed filtered matching network (FMN) aimed to improve the key parameters of the PA which are bandwidth and the output power. The input and output matching networks along with the PA design is analysed for achievement of wide bandwidth and linear output power. Furthermore, the power amplifier linearity performance is evaluated in terms of ACLR. Then an improving bandwidth and output power amplifier would be achieved by optimizing the matching network. A maximized efficiency at extended linear region of power amplifier is allowed by using matching network.

Mobile communication systems operating over a frequency range of $700 \mathrm{MHz}$ to $3500 \mathrm{MHz}$ are standardized for $2 \mathrm{G}$ and beyond LTE/LTE-A. Figure 1 shows the proposed matching network along with PA architecture. The input matching network, is a combination of cascaded LC that is also indicated in Figure 2. The LC network provides an optimized impedance transformation through harmonic suppression of power amplifier's signal. On the contrary, the output matching network is designed using T-section band pass filter architecture which ensures further harmonic suppression and impedance transformation of PA signal. Distortions caused by harmonics are reduced at the input and output matching network by use of filter which shows that harmonic suppression is highly required.

The proposed filtered matching network (FMN) in the PA design is shown in Figure 2. The designed PA allows VDD supply of $3.3 \mathrm{~V}$ to the transistors without exceeding the potential breakdown of transistor's oxide. The transistors are divided into four cells to reduce the parasitic effect and its outputs are combined by means of T-section filters. This architecture results improved ACLR performance as it eliminates the harmonics caused by the PA. Therefore, proposed FMN will provide good matching and improves all the PA parameters such as bandwidth, output power, gain, and ACLR.

\section{A. Design Principles}

The input matching network that includes LC impedance transformation stage is designed for a source impedance, $\mathrm{Zs}=50 \Omega$, with measured PA input impedance, $\mathrm{Z}_{\mathrm{in}, \mathrm{PA}}$ of $2.6-\mathrm{j} 7 \Omega$ using cadence simulation. The input matching network's capacitive and inductive parameters is determined using equations (1) to (5) [33]: 


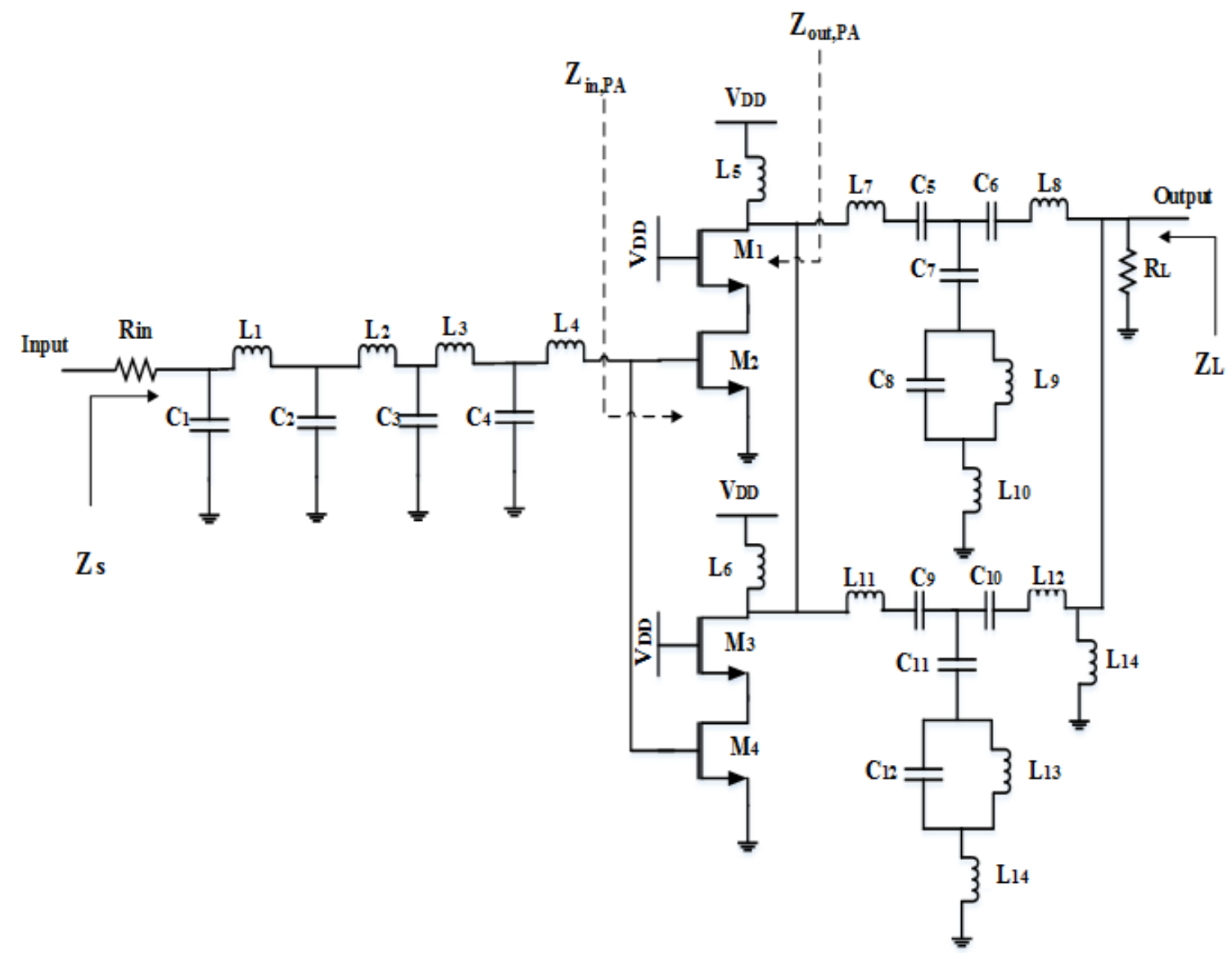

Fig.2.Circuit diagram of wideband PA with proposed matching network

$R_{\text {in }}=\sqrt{\frac{L_{1}}{C_{1}}}$

$f_{c}=\frac{1}{\pi \cdot \sqrt{L_{1} C_{1}}}$

Where $R_{\text {in }}$ of $50 \Omega$ and $f_{c}$ of $2370 \mathrm{MHz}$ from equations (1) and (2) are known as input resistance and carrier frequency. From equations (1) and (2), it can be determined that

$R_{\text {in }} \cdot f_{c}=\frac{1}{\pi \cdot C_{1}}$

The design equation for capacitance $\left(\mathrm{C}_{1}\right)$ and inductance $\left(\mathrm{L}_{1}\right)$ for the LC matching network at input side are expressed as in equation (4) and (5):

$C_{1}=\frac{1}{R_{\text {in }} \cdot \pi \cdot f_{c}}$

$L_{1}=\frac{R_{\text {in }}}{\pi \cdot f_{c}}$

Where equation (4) also applies to the calculation for $\mathrm{C}_{2}, \mathrm{C}_{3}$ and $\mathrm{C}_{4}$. Similarly, equation (5) applies to $\mathrm{L}_{2}, \mathrm{~L}_{3}$ and $\mathrm{L}_{4}$.

Similarly, the output matching network is designed for load impedance, $Z_{L}=50 \Omega$, and the measured PA output impedance, $Z_{\text {out,PA }}$ as $2.2-\mathrm{j} 0.8 \Omega$. The output matching network aspects is derived through equations (6) to (11) [33]:

$C_{5}=\frac{2 C_{5 a}}{m}$ 


$$
\begin{aligned}
& C_{7}=\left(\frac{4 m}{1-m^{2}}\right) \cdot C_{5 a} \\
& C_{8}=m \cdot C_{6 a} \\
& L_{7}=\frac{m \cdot L_{5 a}}{2} \\
& L_{9}=\frac{L_{6 a}}{m} \\
& L_{10}=\left(\frac{1-m^{2}}{4 m}\right) \cdot L_{5 a}
\end{aligned}
$$

Where

$$
\begin{aligned}
C_{5 a} & =\frac{f_{2}-f_{1}}{4 \pi R_{\text {in }} \cdot\left(f_{2} \cdot f_{1}\right)} \\
C_{6 a} & =\frac{1}{\pi R_{\text {in }} \cdot\left(f_{2}-f_{1}\right)} \\
L_{5 a} & =\frac{R_{\text {in }}}{\pi \cdot\left(f_{2}-f_{1}\right)} \\
L_{6 a} & =\frac{R_{i} \cdot\left(f_{2}-f_{1}\right)}{4 \pi \cdot\left(f_{2} \cdot f_{1}\right)}
\end{aligned}
$$

Where $f_{1}$ of $900 \mathrm{MHz}$ is the lower cut-off frequency and $\mathrm{f}_{2}$ of $2370 \mathrm{MHz}$ is the upper cut-off frequency. Also, the coefficient in the $\mathrm{m}$-derived section is represented by $\mathrm{m}$. In addition, the derived equations would ensure that PA is matched to input and output matching network in terms of improved output power and bandwidth.

The layout of the proposed power amplifier is shown Figure 3 below.

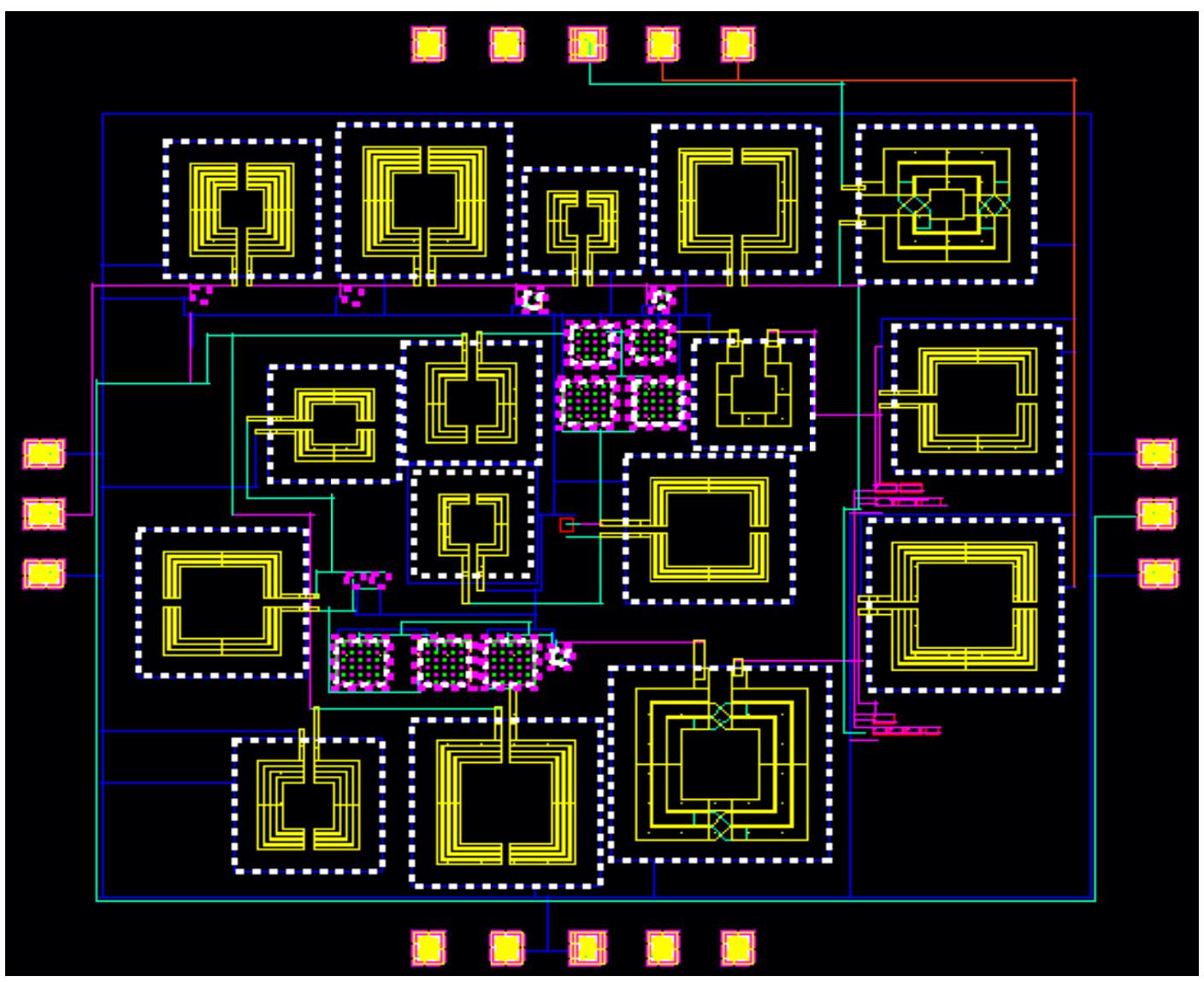

Fig. 3. Layout of proposed power amplifier 


\section{III.RESULTS}

The S-parameter analysis has been conducted to determine the bandwidth performance of the designed PA using cadence simulation and the results are indicated in Figure 4.

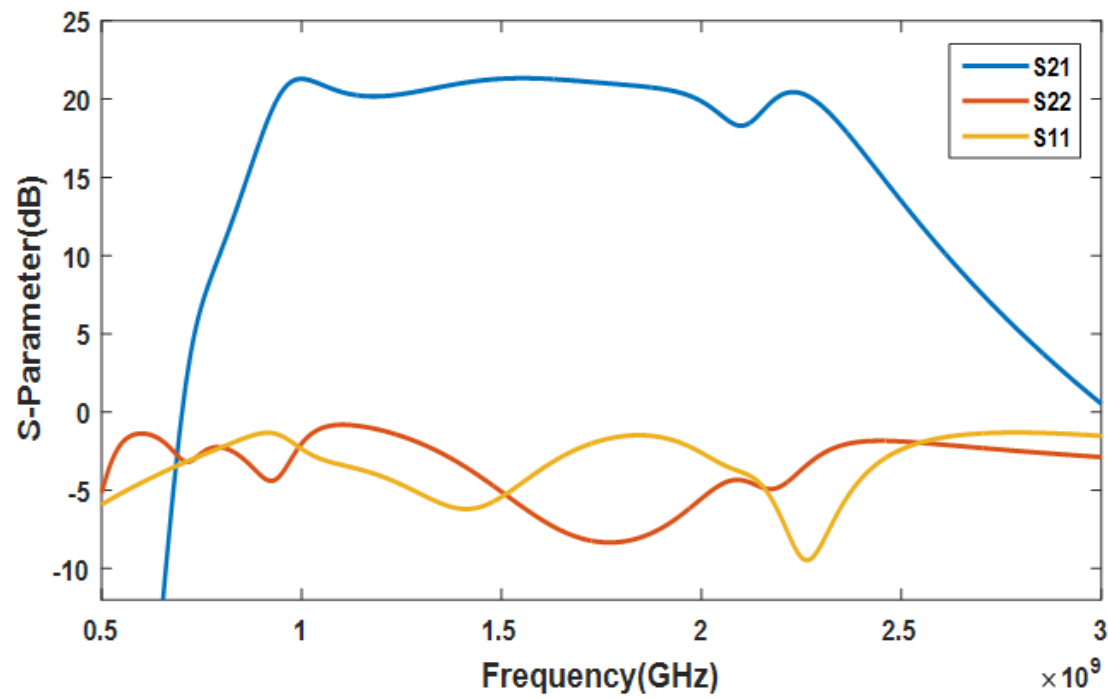

Fig. 4. S-Parameters of wideband PA

The designed PA has wide bandwidth of $1.47 \mathrm{GHz}$ ranging fromfrequency of $900 \mathrm{MHz}$ to $2370 \mathrm{MHz}$ covering 24 LTE bands: 1-4, 8-11, 21, 23-25, 30, 33-37, 39-40, 45, 65-66 \& 70 bands as depicted in Figure 4. Gain represented by S21 shows value of $20.63 \mathrm{~dB}$ as indicated in the S-parameter. The S11 and S22 are reflection coefficients which are below $0 \mathrm{~dB}$ indicating that performance throughout the bandwidth is optimized by matching network.

The periodic steady state analysis implies the performance of output power of the PA is shown in Figure 5.It is clearly evident from the results that the peak output power is $27.5 \mathrm{dBm}$ and the linear output power of $26 \mathrm{dBm}$ at $1-\mathrm{dB}$ compression point. In addition, the results produced by the designed PA clearly complies with LTE specification with output power requirement of $23 \mathrm{dBm}$ [5].

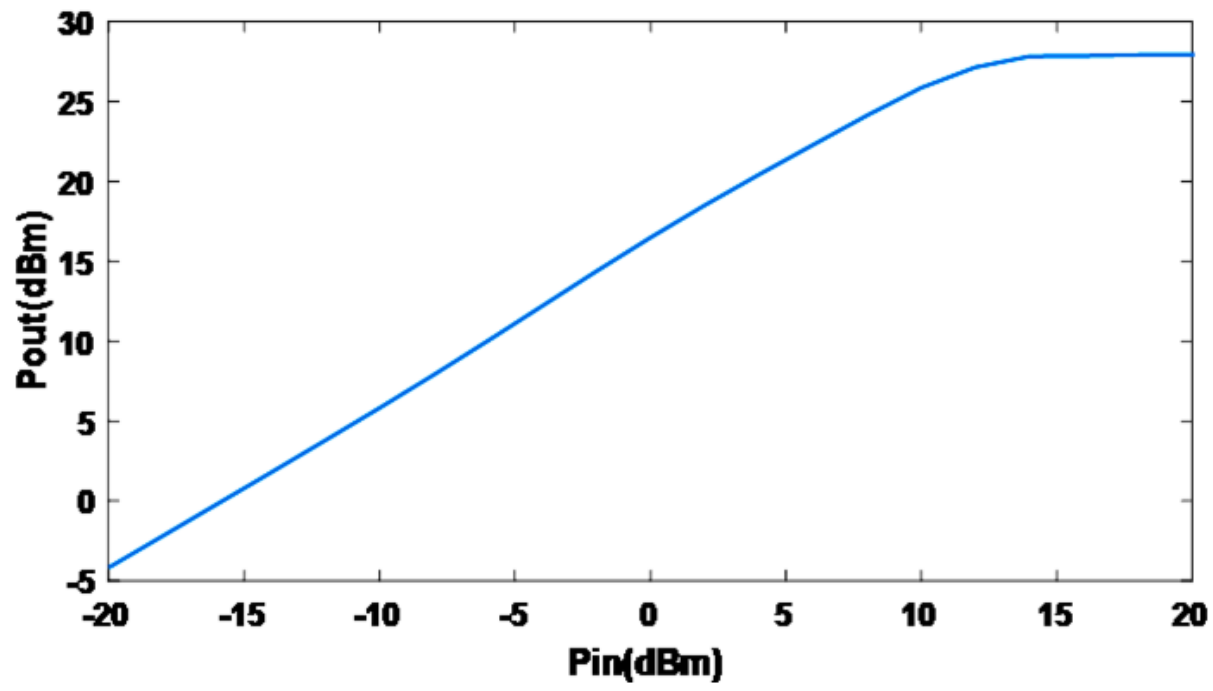

Fig.5. Output power of the wideband PA 


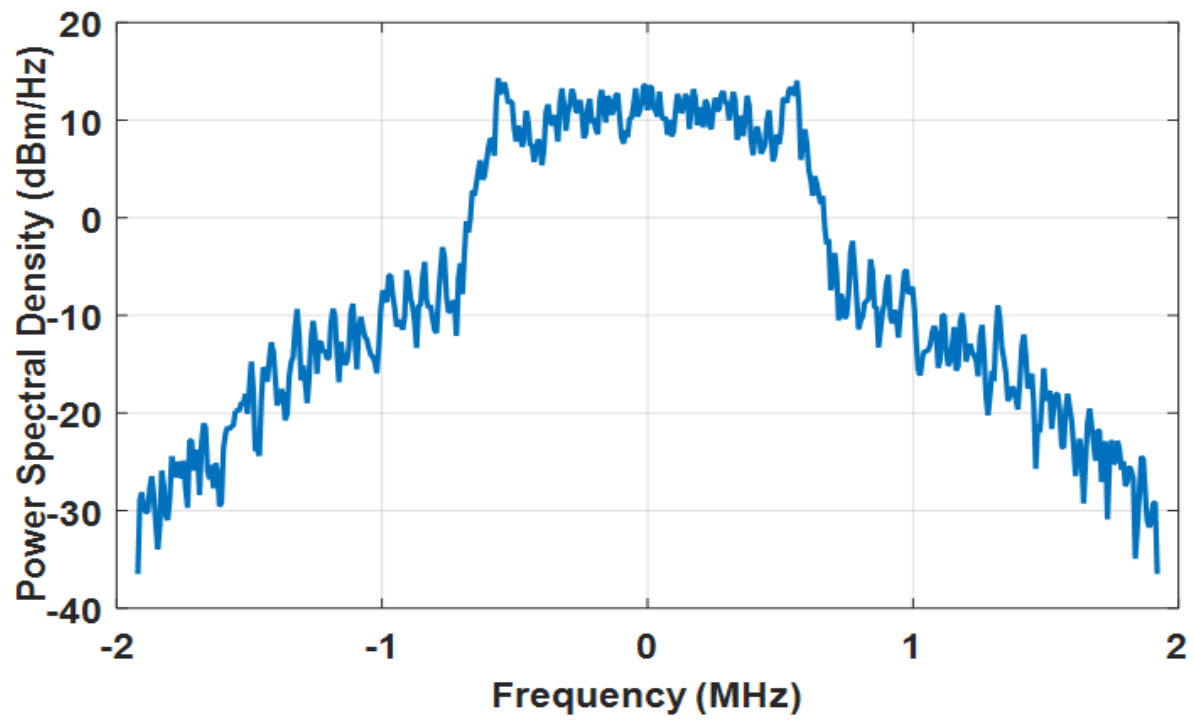

Fig. 6. Output Spectral for LTE

Similarly, Figure 6 indicates that an ACLR -34dBc has been achieved for the PA output power of 27.5dBm which is $1 \mathrm{dBc}$ better than the ACLR requirement of LTE which is $-33 \mathrm{dBc}$ as shown in ACLR analysis of PA design [5]. Table I shows the comparison of the designed wideband PA with state-of-the-art PAs in the literature. The summary of comparison table clearly shows that the designed PA have a reasonable increase in the output power with a wide bandwidth of $1.47 \mathrm{GHz}$ and reduced ACLR of $-34 \mathrm{dBc}$ compared to the literatures highlighted in the table.

TABLE I. Summary of comparison results

\begin{tabular}{|c|c|c|c|c|}
\hline Literature & $\begin{array}{c}\text { Output Power } \\
\text { (Pout), (dBm) }\end{array}$ & $\begin{array}{c}\text { Frequency } \\
\text { range (GHz) }\end{array}$ & $\begin{array}{c}\text { Bandwidth } \\
\text { (GHz) }\end{array}$ & $\begin{array}{c}\text { ACLR } \\
\text { (dBc) }\end{array}$ \\
\hline$[1]$ & 26 & $1.7-1.98$ & 0.28 & -32 \\
\hline$[3]$ & 28 & $1.85-2.5$ & 0.65 & -33 \\
\hline$[9]$ & 27.3 & $1.7-2.5$ & 0.8 & -33 \\
\hline$[14]$ & 24 & $0.75-1.05$ & 0.3 & -45 \\
\hline$[19]$ & 27.1 & $1.6-2.6$ & 1 & - \\
\hline This work & 27.5 & $0.9-2.37$ & 1.47 & -34 \\
\hline
\end{tabular}

\section{IV.CONCLUSION}

In this paper, a wideband PA with filtered matching network (FMN) is proposed, designed and simulated at LTE bands. The results obtained clearly indicate that the PA provides an appreciable gain of $20.63 \mathrm{~dB}$ over the frequency ranging from 900 to $2370 \mathrm{MHz}$ and has an output power of $27.5 \mathrm{dBm}$ with reduced ACLR of $-34 \mathrm{dBc}$. The proposed wideband PA with filtered matching network operates over 24 LTE bands stated in section III. The proposed PA is a feasible solution for the current demand ofwideband PA in mobile communication systems.

\section{REFERENCES}

[1] M. Akbarpour, M. Helaoui, and F. Ghannouchi, "Analytical Design Methodology for Generic Doherty Amplifier Architectures Using Three-Port Input/Output Networks," IEEE, vol. 63, no. 10, pp. 3242-3253, 2015.

[2] M. Lee, C. Liou, W. Tsai, H. Hsu, and S. Mao, "Fully Monolithic BiCMOS Reconfigurable Power Amplifier for Multi-Mode and Multi-Band Applications," IEEE, vol. 62, no. 2, pp. 614-624, 2015.

[3] S. Kang, U. Kim, and J. Kim, "A Multi-Mode Multi-Band Reconfigurable Power Amplifier for 2G/3G/4G Handset Applications," IEEE, vol. 25, no. 1, pp. 49-51, 2015 M. Akbarpour, M. Helaoui, and F. Ghannouchi, "Analytical Design Methodology for Generic Doherty Amplifier Architectures Using Three-Port Input/Output Networks," IEEE, vol. 63, no. 10, pp. 3242-3253, 2015.

[4] G. Lee, J. Jonghun, and J. Song, "A Multiband Power Amplifier With a Reconfigurable Output-Matching network for 10-MHz BW LTE Mobile Phone Applications," IEEE, vol. 62, no. 6, pp. 558-562, 2015.

[5] ETSI, "Evolved Universal Terrestrial Radio Access (E-UTRA) Base Station (BS) radio transmission and reception," ETSI, vol. 14, no. 2, pp. 1-154, 2017.

[6] J. Xia, M. Yang, and A. Zhu, "Improved Doherty Amplifier Design with Minimum Phase Delay in Output Matching network for Wideband Application," IEEE, vol. 26, no. 11, pp. 915-917, 2016. 
[7] M. Rubio, J. Fang, V. Camarchia, R. Quaglia, M. Pirola, and G.Giovanni, "3-3.6-GHz Wideband GaN Power Amplifier Exploiting Output Compensation Stages," IEEE, vol. 60, no. 8, pp. 2543-2548, 2012.

[8] A. Mohamed, S. Boumaiza, and R. Mansour, "Electronically Tunable Power Amplifier for Multi-Mode Multi-Band Base Stations," IEEE, vol. 61, no. 4, pp. 1229-1240, 2014

[9] D. Fritsche, R.Wolf, and F. Ellinger, "Analysis and design of a stacked power amplifier with very high bandwidth," IEEE, vol. 60, no. 10, pp. 3223-3231, Oct. 2012.

[10] C. Mia, S. Shao, C. Qing, and Y. Tang, "A Wideband Power Amplifier With 100 MHz Instantaneous Bandwidth for LTE-Advanced Applications," IEEE, vol. 23, no. 11, pp. 614-616, 2013.

[11] J. Moon, J. Son, J. Lee, and B. Kim, "A Multimode/Multiband Envelope Tracking Transmitter With Broadband Saturated Amplifier," IEEE, vol. 59, no. 12, pp. 3463-3472, 2011.

[12] J. Son, Y. Park, I. Kim, J. Moon, and B. Kim, "Broadband Saturated Power Amplifier With Harmonic Control Circuits," IEEE, vol. 24, no. 3, pp. 185-187, 2014.

[13] A. Cidronali, S. Maddio, N. Giovannelli, and G. Collodi, "Frequency Analysis and Multiline Implementation of Compensated Impedance Inverter for Wideband Doherty High-Power Amplifier Design," IEEE, vol. 64, no. 5, pp. 1359-1371, 2016

[14] V. Vorapipat, C. Levy, and P. Asbeck, "A Wideband Voltage Mode Power Amplifier," in IEEE, 2016.

[15] Y. Sun, X. Zhu, J. Zhai, L. Zhang, and F. Meng, "Highly Efficient Concurrent Power Amplifier With Controllable Modes," IEEE, vol. 63, no. 12, pp. 4051-4060, 2015.

[16] S. Zheng, Z. Liu, Y. Pan, Y. Wu, W. Chan, and Y. Liu, "Bandpass Filtering Power Amplifier With Enhanced Efficiency and Wideband Harmonic Suppression," IEEE, vol. 63, no. 3, pp. 337-346, 2016.

[17] P. Guo, W. Kong, J. Xia, and L. Yang, "Wideband Power Amplifier Using Suitable Peaking Output Matching network," in IEEE, 2013.

[18] M.Younes, O. Hammi, A. Kwan, and F. Ghannouchi, "An Accurate Complexity-Reduced "PLUME" Model for Behavioral Modeling and Digital Predistortion of RF Power Amplifiers," IEEE, vol. 58, no. 4, pp. 1397-1405, 2011.

[19] A. Aref and R. Negra, "A Fully Integrated Adaptive Multiband Multimode Switching-Mode CMOS Power Amplifier Ahmed," IEEE, vol. 60 , no. 8, pp. 2549-2561, 2012.

[20] J. Kim, P. Roblin, D. Chaillot, and Z. Xie, "A Generalized Architecture for the Frequency-Selective Digital Predistortion Linearization Technique," IEEE, vol. 61, no. 1, pp. 596-605, 2013.

[21] M. Nair, R. Giofre, L. Piazzon, and P. Colantonio, "A Comparative Study on Digital Predistortion Techniques for Doherty Amplifier for LTE Applications," IEEE, vol. 1, pp. 1-3, 2014.

[22] H. Lin, T. Liu, Y. Ye, X. Luo, G. Cao, and C. Sun, "Doherty Power Amplifier Linearization with Augmented Hammerstein Model for LTE Systems," IEEE, pp. 1-4, 2012.

[23] O. Hammi, "Augmented Twin-Nonlinear Two-Box Behavioral Models for Multicarrier LTE Power Amplifiers," The Scientific World Journal, vol. 2014, pp. 1-7, 2014.

[24] B. Lin, Y. Wang, B. Feuvrie, and Q. Dai, "A Novel Power Amplifier Linearization Technique based on Pre-distortion Principle and Wiener model," International Journal of Advanced Science and Technology, vol. 22, pp. 59-70, 2010.

[25] K. Niotaki, A. Collado, A. Georgiadis, and J. Vardakas, "5 Watt GaN HEMT Power Amplifier for LTE," RADIOENGINEERING, vol. 23, no. 1, pp. 338-344, 2014.

[26] M. Nair, R. Giofre, L. Piazzon, and P. Colantonio, "An Overview of RF Power Amplifier Digital Predistortion Techniques for Wireless Communication Systems," TUSUR (TУCУPa), vol. 2 (26), no. 2, pp. 152-157, 2012.

[27] Z. Fu, L. Anttila, M. Abdelaziz, M. Valkama, and A. Wyglinski, "Frequency-Selective Digital Predistortion for Unwanted Emission Reduction," IEEE, vol. 63, no. 1, pp. 254-267, 2015.

[28] C. Ma, W. Pan, Y. Tang, and C. Qing, "Design of Unsymmetrical Power Amplifier for 460MHz LTE-Advanced," Atlantis Press, pp. 0407-0410, 2013.

[29] W. Chen, S. Zhang, Y. Liu, F. Ghannouchi, Z. Feng, and Y. Liu, "Efficient Pruning Technique of Memory Polynomial Models Suitable for PA Behavioral Modeling and Digital Predistortion," IEEE, vol. 62, no. 10, pp. 2290-2298, 2014.

[30] J. Valdez, M. Palafox, C. Gontrand, and J. Perez, "Performance Evaluation of a Memory-Polynomial Model for Microwave Power Amplifiers," Programación Matemática y Software, vol. 4, no. 1, pp. 13-23, 2011.

[31] O. Hammi, A. Abdelrahman, A. Kwan, A. Zerguine, and F. Ghannouchi, "A Novel Weighted Memory Polynomial for Behavioral Modeling and Digital Predistortion of Nonlinear Wireless Transmitters," IEEE, vol. 63, no. 3, pp. 1745-1753, 2016.

[32] S. Badhwar, B. Kaur, G. Badhwar, and A. Sappal, "Design of Digital Predistortion Technique for RF Power Amplifier using Memory Polynomial," Int. Journal of Engineering Research and Applications, vol. 4, no. 9, pp. 1-3, 2014.

[33] D.M Pozar, Microwave Engineering, 4th ed. New York, NY, USA: Wiley, 2011 